\title{
On a difference scheme of second order of accuracy for the Bitsadze-Samarskii type nonlocal boundary-value problem
}

\author{
Allaberen Ashyralyev ${ }^{1}$ and Elif Ozturk ${ }^{2 *}$
}

"Correspondence:
eozturk@comu.edu.tr
2Department of Econometrics,
Canakkale Onsekiz Mart University,
Canakkale, 17200, Turkey
Full list of author information is
available at the end of the article

\begin{abstract}
In this study, the Bitsadze-Samarskii type nonlocal boundary-value problem with integral condition for an elliptic differential equation in a Hilbert space $H$ with self-adjoint positive definite operator $A$ is considered. The second order of the accuracy difference scheme for the approximate solutions of this nonlocal boundary-value problem is presented. The well-posedness of this difference scheme in Hölder spaces with a weight is proved. The theoretical statements for the solution of this difference scheme are supported by the results of numerical example.
\end{abstract}

Keywords: well-posedness; difference scheme; elliptic equation

\section{Introduction}

In 1969 Bitsadze and Samarskii [1] stated and studied a new problem in which a nonlocal condition is related to the values of the solution on parts of the boundary and on an interior curve for a uniformly elliptic equation. Furthermore, in [2-16] the Bitsadze-Samarskii type nonlocal boundary-value problems were investigated for the various differential and difference equations of elliptic type. The role played by coercive inequalities in the study of local boundary-value problems for elliptic differential equations is well known [17]. Methods of solutions of elliptic differential and difference equations have been studied extensively by many researchers (see [18-27] and the references therein). In the present paper we consider the Bitsadze-Samarskii type nonlocal boundary-value problem with integral condition,

$$
\left\{\begin{array}{l}
-\frac{d^{2} u(t)}{d t^{2}}+A u(t)=f(t), \quad 0<t<1, \\
u(0)=\varphi, \quad u(1)=\int_{0}^{1} \rho(\lambda) u(\lambda) d \lambda+\psi
\end{array}\right.
$$

for the differential equation of elliptic type in a Hilbert space $H$ with the self-adjoint positive definite operator $A$ with a closed domain $D(A) \subset H$. Here, let $f(t)$ be a given abstract continuous function defined on $[0,1]$ with values in $H, \varphi$, and $\psi$ are elements of $D(A)$ and $\rho(t)$ is a scalar function. A function $u(t)$ is called a solution of problem (1) if the following conditions are satisfied:

i. $u(t)$ is a twice continuously differentiable on the segment $[0,1]$.

ii. The element $u(t)$ belongs to $D(A)$ for all $t \in[0,1]$, and the function $A u(t)$ is continuous on the segment $[0,1]$.

iii. $u(t)$ satisfies the equation and nonlocal boundary conditions (1). 
The paper is organized as follows. In Section 2 the second order of the accuracy difference scheme for the approximate solution (1) is presented. The stability, the almost coercive stability, and the coercive stability estimates for the solution of the difference scheme for an approximate solution of the nonlocal boundary-value problem with integral condition for elliptic equations are obtained. Section 3 contains the applications of Section 2. The final section is devoted to the numerical result. Theoretical statements for the solution of the second order of the accuracy difference scheme is supported by a numerical experiment.

\section{The second order of the accuracy difference scheme}

Let us associate the nonlocal boundary-value problem (1) with the corresponding difference problem,

$$
\left\{\begin{array}{c}
-\frac{1}{\tau^{2}}\left[u_{k+1}-2 u_{k}+u_{k-1}\right]+A u_{k}=\varphi_{k} \\
\varphi_{k}=f\left(t_{k}\right), t_{k}=k \tau, 1 \leq k \leq N-1, N \tau=1 \\
u_{0}=\varphi, \quad u_{N}=\sum_{j=1}^{N} \rho\left(t_{j}-\frac{\tau}{2}\right)\left(\frac{u_{j}+u_{j-1}}{2}\right) \tau+\psi
\end{array}\right.
$$

We will study the problem (2) under the following assumption:

$$
\sum_{j=1}^{N}\left|\rho\left(t_{j}-\frac{\tau}{2}\right)\right| \tau<1
$$

It is well known [28] that for a self-adjoint positive definite operator $A$ it follows that $B=$ $\frac{1}{2}\left(\tau A+\sqrt{4 A+\tau^{2} A^{2}}\right)$ is self-adjoint positive definite and $R=(I+\tau B)^{-1}$, which is defined on the whole space $H$ is a bounded operator. Here, $I$ is the unit operator. Furthermore, we have

$$
\left\{\begin{array}{l}
\left\|\left(I-R^{2 N}\right)^{-1}\right\|_{H \rightarrow H} \leq M(\delta), \\
\left\|R^{k}\right\|_{H \rightarrow H} \leq M(\delta)(1+\delta \tau)^{-k}, \\
k \tau\left\|B R^{k}\right\|_{H \rightarrow H} \leq M(\delta), \quad k \geq 1, \delta>0, \\
\left\|B^{\beta}\left(R^{k+r}-R^{k}\right)\right\|_{H \rightarrow H} \leq M(\delta) \frac{(r \tau)^{\alpha}}{(k \tau)^{\alpha+\beta}}, \quad 1 \leq k<k+r \leq N, 0 \leq \alpha, \beta \leq 1 .
\end{array}\right.
$$

In this paper, positive constants, which can differ in time (hence they are not a subject of precision considerations) will be indicated with $M$. On the other hand $M(\alpha, \beta, \ldots)$ is used to focus on the fact that the constant depends only on $\alpha, \beta, \ldots$.

Lemma 1 The operator

$$
I-\sum_{j=1}^{N} \rho\left(t_{j}-\frac{\tau}{2}\right) \frac{\tau}{2}\left(I-R^{2 N}\right)^{-1}\left(R^{N-j}-R^{N+j}+R^{N-j+1}-R^{N+j-1}\right)
$$

has an inverse

$$
S_{\tau}=\left(I-\sum_{j=1}^{N} \rho\left(t_{j}-\frac{\tau}{2}\right) \frac{\tau}{2}\left(I-R^{2 N}\right)^{-1}\left(R^{N-j}-R^{N+j}+R^{N-j+1}-R^{N+j-1}\right)\right)^{-1}
$$


and the following estimate is satisfied:

$$
\left\|S_{\tau}\right\|_{H \rightarrow H} \leq M(\delta) \tau
$$

where $M$ does not depend on $\tau$.

The proof of the estimate (5) is based on the estimate

$$
\langle(I-L) u, u\rangle \geq\left(1-\sum_{j=1}^{N} \rho\left(t_{j}-\frac{\tau}{2}\right) \tau\right)\langle u, u\rangle .
$$

Here

$$
L=\sum_{j=1}^{N} \rho\left(t_{j}-\frac{\tau}{2}\right) \frac{\tau}{2}\left(I-R^{2 N}\right)^{-1}\left(R^{N-j}-R^{N+j}+R^{N-j+1}-R^{N+j-1}\right) .
$$

The estimate (6) follows from the spectral representation of $A$ and the Cauchy inequality.

Theorem 2 For any $\varphi_{k}, 1 \leq k \leq N-1$, the solution of the problem (2) exists and the following formula holds:

$$
\begin{aligned}
u_{k}= & \left(I-R^{2 N}\right)^{-1}\left\{\left(R^{k}-R^{2 N-k}\right) \varphi+\left(R^{N-k}-R^{N+k}\right) u_{N}\right. \\
& \left.-\left(R^{N-k}-R^{N+k}\right)(I+\tau B)(2 I+\tau B)^{-1} B^{-1} \sum_{i=1}^{N-1}\left(R^{N-1-i}-R^{N-1+i}\right) \varphi_{i} \tau\right\} \\
& +(I+\tau B)(2 I+\tau B)^{-1} B^{-1} \sum_{i=1}^{N-1}\left(R^{|k-i|-1}-R^{k+i-1}\right) \varphi_{i} \tau
\end{aligned}
$$

for $k=0, \ldots, N-1$,

$$
\begin{aligned}
u_{N}= & S_{\tau}\left(\sum _ { j = 1 } ^ { N } \rho ( t _ { j } - \frac { \tau } { 2 } ) \frac { \tau } { 2 } \left[\left\{\left(I-R^{2 N}\right)^{-1}\left(R^{j}-R^{2 N-j}+R^{j-1}-R^{2 N-j+1}\right) \varphi\right.\right.\right. \\
& -\left(R^{N-j}-R^{N+j}+R^{N-j+1}-R^{N+j-1}\right)(I+\tau B)(2 I+\tau B)^{-1} \\
& \left.\times \sum_{i=1}^{N-1} B^{-1}\left(R^{N-1-i}-R^{N-1+i}\right) \varphi_{i} \tau\right\}+(I+\tau B)(2 I+\tau B)^{-1} B^{-1} \\
& \left.\left.\times \sum_{i=1}^{N-1}\left(R^{|j-i|-1}-R^{j+i-1}+R^{|j-1-i|-1}-R^{j+i-2}\right) \varphi_{i} \tau\right]+\psi\right)
\end{aligned}
$$

for $k=N$.

Proof

$$
\left\{\begin{array}{l}
-\frac{1}{\tau^{2}}\left[u_{k+1}-2 u_{k}+u_{k-1}\right]+A u_{k}=\varphi_{k}, \quad 1 \leq k \leq N-1, N \tau=1, \\
u_{0}=\varphi, u_{N} \text { are given }
\end{array}\right.
$$


has a solution and the following formula holds [29]:

$$
\begin{aligned}
u_{k}= & \left(I-R^{2 N}\right)^{-1}\left\{\left(R^{k}-R^{2 N-k}\right) \varphi+\left(R^{N-k}-R^{N+k}\right) u_{N}\right. \\
& \left.-\left(R^{N-k}-R^{N+k}\right)(I+\tau B)(2 I+\tau B)^{-1} B^{-1} \sum_{i=1}^{N-1}\left(R^{N-1-i}-R^{N-1+i}\right) \varphi_{i} \tau\right\} \\
& +(I+\tau B)(2 I+\tau B)^{-1} B^{-1} \sum_{i=1}^{N-1}\left(R^{|k-i|-1}-R^{k+i-1}\right) \varphi_{i} \tau .
\end{aligned}
$$

Applying formula (9) and the nonlocal boundary condition

$$
u_{N}=\sum_{j=1}^{N} \rho\left(t_{j}-\frac{\tau}{2}\right)\left(\frac{u_{j}+u_{j-1}}{2}\right) \tau+\psi,
$$

we obtain

$$
\begin{aligned}
u_{N}= & \left(I-\sum_{j=1}^{N} \rho\left(t_{j}-\frac{\tau}{2}\right) \frac{\tau}{2}\left(I-R^{2 N}\right)^{-1}\left(R^{N-j}-R^{N+j}+R^{N-j+1}-R^{N+j-1}\right)\right)^{-1} \\
& \times\left(\sum _ { j = 1 } ^ { N } \rho ( t _ { j } - \frac { \tau } { 2 } ) \frac { \tau } { 2 } \left[( I - R ^ { 2 N } ) ^ { - 1 } \left\{\left(R^{j}-R^{2 N-j}+R^{j-1}-R^{2 N-j+1}\right) \varphi\right.\right.\right. \\
& -\left(R^{N-j}-R^{N+j}+R^{N-j+1}-R^{N+j-1}\right) \\
& \left.\times(I+\tau B)(2 I+\tau B)^{-1} B^{-1} \sum_{i=1}^{N-1}\left(R^{N-1-i}-R^{N-1+i}\right) \varphi_{i} \tau\right\}+(I+\tau B)(2 I+\tau B)^{-1} B^{-1} \\
& \left.\left.\times \sum_{i=1}^{N-1}\left(R^{|j-i|-1}-R^{j+i-1}+R^{|j-1-i|-1}-R^{j+i-2}\right) \varphi_{i} \tau\right]+\psi\right) .
\end{aligned}
$$

Since the operator

$$
I-\sum_{j=1}^{N} \rho\left(t_{j}-\frac{\tau}{2}\right) \frac{\tau}{2}\left(I-R^{2 N}\right)^{-1}\left(R^{N-j}-R^{N+j}+R^{N-j+1}-R^{N+j-1}\right)
$$

has an inverse $S_{\tau}$, it follows that

$$
\begin{aligned}
u_{N}= & S_{\tau}\left(\sum _ { j = 1 } ^ { N } \rho ( t _ { j } - \frac { \tau } { 2 } ) \frac { \tau } { 2 } \left[( I - R ^ { 2 N } ) ^ { - 1 } \left\{\left(R^{j}-R^{2 N-j}+R^{j-1}-R^{2 N-j+1}\right) \varphi\right.\right.\right. \\
& -\left(R^{N-j}-R^{N+j}+R^{N-j+1}-R^{N+j-1}\right) \\
& \left.\times(I+\tau B)(2 I+\tau B)^{-1} B^{-1} \sum_{i=1}^{N-1}\left(R^{N-1-i}-R^{N-1+i}\right) \varphi_{i} \tau\right\}+(I+\tau B)(2 I+\tau B)^{-1} B^{-1} \\
& \left.\left.\times \sum_{i=1}^{N-1}\left(R^{|j-i|-1}-R^{j+i-1}+R^{|j-1-i|-1}-R^{j+i-2}\right) \varphi_{i} \tau\right]+\psi\right) .
\end{aligned}
$$

Theorem 2 is proved. 
Let $\mathcal{F}\left([0,1]_{\tau}, H\right)$ be the linear space of the mesh functions $\varphi^{\tau}=\left\{\varphi_{k}\right\}_{1}^{N-1}$ with values in the Hilbert space $H$. We denote by $C\left([0,1]_{\tau}, H\right)$ and $C_{01}^{\alpha}\left([0,1]_{\tau}, H\right), 0<\alpha<1$, Banach spaces with the norms

$$
\begin{aligned}
\left\|\varphi^{\tau}\right\|_{C\left([0,1]_{\tau}, H\right)}= & \max _{1 \leq k \leq N-1}\left\|\varphi_{k}\right\|_{H}, \\
\left\|\varphi^{\tau}\right\|_{C_{01}^{\alpha}\left([0,1]_{\tau}, H\right)}= & \left\|\varphi^{\tau}\right\|_{C\left([0,1]_{\tau}, H\right)} \\
& +\sup _{1 \leq k \leq k+r \leq N-1} \frac{((N-k) \tau)^{\alpha}((k+r) \tau)^{\alpha}}{(r \tau)^{\alpha}}\left\|\varphi_{k+r}-\varphi_{k}\right\|_{H} .
\end{aligned}
$$

The nonlocal boundary-value problem (2) is said to be stable in $\mathcal{F}\left([0,1]_{\tau}, H\right)$ if we have the inequality

$$
\left\|u^{\tau}\right\|_{\mathcal{F}\left([0,1]_{\tau}, H\right)} \leq M(\delta)\left[\left\|\varphi^{\tau}\right\|_{\mathcal{F}\left([0,1]_{\tau}, H\right)}+\|\varphi\|_{H}+\|\psi\|_{H}\right] .
$$

Theorem 3 The solutions of the difference scheme (2) under the assumption (3) satisfy the stability estimate

$$
\left\|u^{\tau}\right\|_{C\left([0,1]_{\tau}, H\right)} \leq M(\delta)\left[\left\|\varphi^{\tau}\right\|_{C\left([0,1]_{\tau}, H\right)}+\|\varphi\|_{H}+\|\psi\|_{H}\right] .
$$

Proof By [29],

$$
\left\|u^{\tau}\right\|_{C\left([0,1]_{\tau}, H\right)} \leq M(\delta)\left[\left\|\varphi^{\tau}\right\|_{C\left([0,1]_{\tau}, H\right)}+\|\varphi\|_{H}+\left\|u_{N}\right\|_{H}\right]
$$

is proved for the solution of difference scheme (8). Then the proof of (10) is based on (11) and on the estimate

$$
\left\|u_{N}\right\|_{H} \leq M(\delta)\left[\left\|\varphi^{\tau}\right\|_{C\left([0,1]_{\tau}, H\right)}+\|\varphi\|_{H}+\|\psi\|_{H}\right] .
$$

Using the formula (7) and the estimates (4), (5), we get

$$
\begin{aligned}
\left\|u_{N}\right\|_{H} \leq & \left\|S_{\tau}\right\|_{H \rightarrow H}\left(\sum _ { j = 1 } ^ { N } | \rho ( t _ { j } - \frac { \tau } { 2 } ) | \frac { \tau } { 2 } \left[\| ( I - R ^ { 2 N } ) ^ { - 1 } \| _ { H \rightarrow H } \left\{\left(\left\|R^{j}\right\|_{H \rightarrow H}\right.\right.\right.\right. \\
& \left.+\left\|R^{2 N-j}\right\|_{H \rightarrow H}+\left\|R^{j-1}\right\|_{H \rightarrow H}+\left\|R^{2 N-j+1}\right\|_{H \rightarrow H}\right)\|\varphi\|_{H}+\left(\left\|R^{N-j}\right\|_{H \rightarrow H}\right. \\
& \left.+\left\|R^{N+j}\right\|_{H \rightarrow H}+\left\|R^{N-j+1}\right\|_{H \rightarrow H}+\left\|R^{N+j-1}\right\|_{H \rightarrow H}\right)\left\|(I+\tau B)(2 I+\tau B)^{-1}\right\|_{H \rightarrow H} \\
& \left.\times\left\|B^{-1}\right\|_{H \rightarrow H} \sum_{i=1}^{N-1} \tau\left(\left\|R^{N-i-1}\right\|_{H \rightarrow H}+\left\|R^{N+i-1}\right\|_{H \rightarrow H}\right)\left\|\varphi_{i}\right\|_{H}\right\} \\
& +\left\|(I+\tau B)(2 I+\tau B)^{-1}\right\|_{H \rightarrow H}\left\|B^{-1}\right\|_{H \rightarrow H} \\
& \times\left(\sum_{i=1}^{j-1} \tau\left\|R^{j-i-1}\right\|_{H \rightarrow H}\left\|\varphi_{i}\right\|_{H}+\sum_{i=1}^{j-1} \tau\left\|R^{j-i-2}\right\|_{H \rightarrow H}\left\|\varphi_{i}\right\|_{H}\right. \\
& +\sum_{i=j}^{N-1} \tau\left\|R^{i-j-2}\right\|_{H \rightarrow H}\left\|\varphi_{i}\right\|_{H}+\sum_{i=j}^{N-1} \tau\left\|R^{i-j-1}\right\|_{H \rightarrow H}\left\|\varphi_{i}\right\|_{H}
\end{aligned}
$$




$$
\begin{aligned}
& \left.\left.\left.\quad+\sum_{i=1}^{N-1} \tau\left\|R^{j+i-1}\right\|_{H \rightarrow H}\left\|\varphi_{i}\right\|_{H}+\sum_{i=1}^{N-1} \tau\left\|R^{j+i-2}\right\|_{H \rightarrow H}\left\|\varphi_{i}\right\|_{H}\right)\right]+\|\psi\|_{H}\right) \\
& \leq M(\delta)\left[\left\|\varphi^{\tau}\right\|_{C\left([0,1]_{\tau}, H\right)}+\|\varphi\|_{H}+\|\psi\|_{H}\right] .
\end{aligned}
$$

Theorem 3 is proved.

Theorem 4 The solutions of the difference problem (2) in $C\left([0,1]_{\tau}, H\right)$ under the assumption (3) obey the almost coercive inequality

$$
\begin{aligned}
& \left\|\left\{\tau^{-2}\left(u_{k+1}-2 u_{k}+u_{k-1}\right)\right\}_{1}^{N-1}\right\|_{C\left([0,1]_{\tau}, H\right)}+\left\|\left\{A u_{k}\right\}_{1}^{N}\right\|_{C\left([0,1]_{\tau}, H\right)} \\
& \quad \leq M(\delta)\left[\min \left\{\ln \frac{1}{\tau}, 1+\left|\ln \|B\|_{H \rightarrow H}\right|\right\}\left\|\varphi^{\tau}\right\|_{C\left([0,1]_{\tau}, H\right)}+\|A \varphi\|_{H}+\|A \psi\|_{H}\right] .
\end{aligned}
$$

Proof By [29],

$$
\begin{aligned}
& \left\|\left\{\tau^{-2}\left(u_{k+1}-2 u_{k}+u_{k-1}\right)\right\}_{1}^{N-1}\right\|_{C\left([0,1]_{\tau}, H\right)}+\left\|\left\{A u_{k}\right\}_{1}^{N}\right\|_{C\left([0,1]_{\tau}, H\right)} \\
& \quad \leq M(\delta)\left[\min \left\{\ln \frac{1}{\tau}, 1+\left|\ln \|B\|_{H \rightarrow H}\right|\right\}\left\|\varphi^{\tau}\right\|_{C\left([0,]_{\tau}, H\right)}+\|A \varphi\|_{H}+\left\|A u_{N}\right\|_{H}\right]
\end{aligned}
$$

is proved for the solution of the boundary-value problem (8). Using the estimates (4), (5) and the formula (7), we obtain

$$
\begin{aligned}
& \left\|A u_{N}\right\|_{H} \\
& \quad \leq M(\delta)\left(\min \left\{\ln \frac{1}{\tau}, 1+\left|\ln \|B\|_{H \rightarrow H}\right|\right\}\left\|\varphi^{\tau}\right\|_{C\left([0,1]_{\tau}, H\right)}+\|A \varphi\|_{H}+\|A \psi\|_{H}\right)
\end{aligned}
$$

for the solution of difference scheme (2). Applying formula (7) and $A=B^{2} R$, we get

$$
A u_{N}=J_{1}+J_{2},
$$

where

$$
\begin{aligned}
J_{1}= & S_{\tau}\left(\sum_{j=1}^{N} \rho\left(t_{j}-\frac{\tau}{2}\right) \frac{\tau}{2}\left(I-R^{2 N}\right)^{-1}\left(R^{j}-R^{2 N-j}+R^{j-1}-R^{2 N-j+1}\right) A \varphi+A \psi\right), \\
J_{2}= & S_{\tau}\left(\sum _ { j = 1 } ^ { N } \rho ( t _ { j } - \frac { \tau } { 2 } ) \frac { \tau } { 2 } \left[( I - R ^ { 2 N } ) ^ { - 1 } \left\{-\left(R^{N-j}-R^{N+j}+R^{N-j+1}-R^{N+j-1}\right)(I+\tau B)\right.\right.\right. \\
& \left.\times(2 I+\tau B)^{-1} \sum_{i=1}^{N-1} B\left(R^{N-i}-R^{N+i}\right) \varphi_{i} \tau\right\}+(I+\tau B)(2 I+\tau B)^{-1} \\
& \times \sum_{i=1}^{j-1} B\left(R^{j-i}-R^{j+i}+R^{j-i-1}-R^{j+i-1}\right) \varphi_{i} \tau+(I+\tau B)(2 I+\tau B)^{-1} \\
& \left.\left.\times\left(\sum_{i=j}^{N-1} B\left(R^{i-j}-R^{j+i}+R^{i-j-1}-R^{j+i-1}\right) \varphi_{i} \tau\right)\right]\right) .
\end{aligned}
$$


To this end it suffices to show that

$$
\left\|J_{1}\right\|_{H} \leq M(\delta)\left[\|A \varphi\|_{H}+\|A \psi\|_{H}\right]
$$

and

$$
\left\|J_{2}\right\|_{H} \leq M(\delta) \min \left\{\ln \frac{1}{\tau}, 1+\left|\ln \|B\|_{H \rightarrow H}\right|\right\}\left\|\varphi^{\tau}\right\|_{C\left([0,1]_{\tau}, H\right)} .
$$

The estimate (15) follows from formula (13) and the estimates (4), (5). Using formula (14) and the estimates (4), (5), we obtain

$$
\begin{aligned}
\left\|J_{2}\right\|_{H} \leq & \left\|S_{\tau}\right\|_{H \rightarrow H}\left(\sum_{j=1}^{N}\left|\rho\left(t_{j}-\frac{\tau}{2}\right)\right| \frac{\tau}{2}\right. \\
& \times\left[\| ( I - R ^ { 2 N } ) ^ { - 1 } \| _ { H \rightarrow H } \left\{\left(\left\|R^{N-j}\right\|_{H \rightarrow H}+\left\|R^{N+j}\right\|_{H \rightarrow H}\right.\right.\right. \\
& \left.+\left\|R^{N-j+1}\right\|_{H \rightarrow H}+\left\|R^{N+j-1}\right\|_{H \rightarrow H}\right)\left\|(I+\tau B)(2 I+\tau B)^{-1}\right\|_{H \rightarrow H} \\
& \left.\times \sum_{i=1}^{N-1}\left(\left\|(I-R) R^{N-i-1}\right\|_{H \rightarrow H}+\left\|(I-R) R^{N+i-1}\right\|_{H \rightarrow H}\right)\left\|\varphi_{i}\right\|_{H}\right\} \\
& +\left\|(I+\tau B)(2 I+\tau B)^{-1}\right\|_{H \rightarrow H} \\
& \times\left(\sum_{i=1}^{j}\left\|(I-R) R^{j-i-1}\right\|_{H \rightarrow H}\left\|\varphi_{i}\right\|_{H}+\sum_{i=1}^{j}\left\|(I-R) R^{i+j-1}\right\|_{H \rightarrow H}\left\|\varphi_{i}\right\|_{H}\right. \\
& \left.+\sum_{i=1}^{j}\left\|(I-R) R^{j-i-2}\right\|_{H \rightarrow H}\left\|\varphi_{i}\right\|_{H}+\sum_{i=1}^{j}\left\|(I-R) R^{i+j-2}\right\|_{H \rightarrow H}\left\|\varphi_{i}\right\|_{H}\right) \\
& +\left\|(I+\tau B)(2 I+\tau B)^{-1}\right\|_{H \rightarrow H} \\
& \times\left(\sum_{i=j+1}^{N-1}\left\|(I-R) R^{i-j-1}\right\|_{H \rightarrow H}\left\|\varphi_{i}\right\|_{H}+\sum_{i=j+1}^{N-1}\left\|(I-R) R^{i+j-1}\right\|_{H \rightarrow H}\left\|\varphi_{i}\right\|_{H}\right. \\
& \left.\left.\left.+\sum_{i=j+1}^{N-1}\left\|(I-R) R^{i-j-2}\right\|_{H \rightarrow H}\left\|\varphi_{i}\right\|_{H}+\sum_{i=j+1}^{N-1}\left\|(I-R) R^{i+j-2}\right\|_{H \rightarrow H}\left\|\varphi_{i}\right\|_{H}\right)\right]\right) \\
\leq & M(\delta) \min \left\{\ln \frac{1}{\tau}, 1+\left|\ln \|B\|_{H \rightarrow H}\right|\right\}\left\|\varphi^{\tau}\right\|_{C\left([0,1]_{\tau}, H\right)} \cdot
\end{aligned}
$$

From the last estimate and the estimate (15) follows the estimate (12). Theorem 4 is proved.

Theorem 5 The difference problem (2) is well posed in the Hölder spaces $C_{01}^{\alpha}\left([0,1]_{\tau}, H\right)$ under the assumption (3) and the following coercivity inequality holds:

$$
\begin{aligned}
& \left\|\left\{\tau^{-2}\left(u_{k+1}-2 u_{k}+u_{k-1}\right)\right\}_{1}^{N-1}\right\|_{C_{01}^{\alpha}\left([0,1]_{\tau}, H\right)}+\left\|\left\{A u_{k}\right\}_{1}^{N}\right\|_{C_{01}^{\alpha}\left([0,1]_{\tau}, H\right)} \\
& \quad \leq M(\delta)\left[\frac{1}{\alpha(1-\alpha)}\left\|\varphi^{\tau}\right\|_{C_{01}^{\alpha}\left([0,1]_{\tau}, H\right)}+\|A \varphi\|_{H}+\|A \psi\|_{H}\right] .
\end{aligned}
$$


Proof By [29],

$$
\begin{gathered}
\left\|\left\{\tau^{-2}\left(u_{k+1}-2 u_{k}+u_{k-1}\right)\right\}_{1}^{N-1}\right\|_{C_{01}^{\alpha}\left([0,1]_{\tau}, H\right)}+\left\|\left\{A u_{k}\right\}_{1}^{N-1}\right\|_{C_{01}^{\alpha}\left([0,1]_{\tau}, H\right)} \\
\leq M(\delta) \frac{1}{\alpha(1-\alpha)}\left\|\varphi^{\tau}\right\|_{C_{01}^{\alpha}\left([0,1]_{\tau}, H\right)}+M(\delta)\left[\|A \varphi\|_{H}+\left\|A u_{N}\right\|_{H}\right]
\end{gathered}
$$

is proved for the solution of difference scheme (8). Then the proof of (17) is based on (18) and on the estimate

$$
\left\|A u_{N}\right\|_{H} \leq M(\delta) \frac{1}{\alpha(1-\alpha)}\left\|\varphi^{\tau}\right\|_{C_{01}^{\alpha}\left([0,1]_{\tau}, H\right)}+M(\delta)\left[\|A \varphi\|_{H}+\|A \psi\|_{H}\right] .
$$

Applying the triangle inequality, formula (7) and the estimate (15), we get

$$
\left\|A u_{N}\right\|_{H} \leq\left\|J_{1}\right\|_{H}+\left\|J_{2}\right\|_{H} \leq\left\|J_{2}\right\|_{H}+M(\delta)\left[\|A \varphi\|_{H}+\|A \psi\|_{H}\right] .
$$

To this end it suffices to show that

$$
\left\|J_{2}\right\|_{H} \leq M(\delta) \frac{1}{\alpha(1-\alpha)}\left\|\varphi^{\tau}\right\|_{C_{01}^{\alpha}\left([0,1]_{\tau}, H\right)} .
$$

Applying formula (14), we get

$$
\begin{aligned}
J_{2}= & S_{\tau} \sum_{j=1}^{N} \rho\left(t_{j}-\frac{\tau}{2}\right) \frac{\tau}{2} \\
& \times\left(I-R^{2 N}\right)^{-1}\left\{-\left(R^{N-j}-R^{N+j}+R^{N-j+1}-R^{N+j-1}\right) \tau^{-2}(I-R)^{2}\right. \\
& \times \sum_{i=1}^{j-1} \tau^{2}\left(R^{N-i}-R^{N+i}\right)\left(I-R^{2}\right)^{-1}\left(\varphi_{i}-\varphi_{j}\right) \\
& +\left(-\left(R^{N-j}-R^{N+j}+R^{N-j+1}-R^{N+j-1}\right)\right) \\
& \times \tau^{-2}(I-R)^{2} \sum_{i=j+1}^{N-1} \tau^{2}\left(R^{N-i}-R^{N+i}\right)\left(I-R^{2}\right)^{-1}\left(\varphi_{i}-\varphi_{j}\right) \\
& +\left(I-R^{2 N}\right) \tau^{-2}(I-R)^{2} \sum_{i=1}^{j-1} \tau^{2}\left(R^{j-i}-R^{j+i}+R^{j-i-1}-R^{j+i-1}\right) \\
& \times\left(I-R^{2}\right)^{-1}\left(\varphi_{i}-\varphi_{j}\right)+\left(I-R^{2 N}\right) \tau^{-2}(I-R)^{2} \\
& \times \sum_{i=j+1}^{N-1} \tau^{2}\left(R^{i-j}-R^{j+i}+R^{j-i-1}-R^{j+i-1}\right)\left(I-R^{2}\right)^{-1}\left(\varphi_{i}-\varphi_{j}\right) \\
& -\left(R^{N-j}-R^{N+j}+R^{N-j+1}-R^{N+j-1}\right) \tau^{-2}(I-R)^{2} \\
& \times \sum_{i=1}^{j-1} \tau^{2}\left(R^{N-i}-R^{N+i}\right)\left(I-R^{2}\right)^{-1} \varphi_{j}-\tau^{-2}(I-R)^{2} \\
& \left(R^{N-j}-R^{N+j}+R^{N-j+1}-R^{N+j-1}\right) \sum_{i=j+1}^{N-1} \tau^{2}\left(R^{N-i}-R^{N+i}\right)\left(I-R^{2}\right)^{-1} \varphi_{j} \\
&
\end{aligned}
$$




$$
\begin{aligned}
& +\left(I-R^{2 N}\right) \tau^{-2}(I-R)^{2} \sum_{i=1}^{j-1} \tau^{2}\left(R^{j-i}-R^{j+i}+R^{j-i-1}-R^{j+i-1}\right)\left(I-R^{2}\right)^{-1} \varphi_{j} \\
& \left.+\left(I-R^{2 N}\right) \tau^{-2}(I-R)^{2} \sum_{i=j+1}^{N-1} \tau^{2}\left(R^{i-j}-R^{j+i}+R^{j-i-1}-R^{j+i-1}\right)\left(I-R^{2}\right)^{-1} \varphi_{j}\right\} \\
& =\sum_{z=2}^{4} J_{2}^{z},
\end{aligned}
$$

where

$$
\begin{aligned}
J_{2}^{2}= & S_{\tau} \sum_{j=1}^{N} \rho\left(t_{j}-\frac{\tau}{2}\right) \frac{\tau}{2}\left(I-R^{2 N}\right)^{-1}(I-R)(I+R)^{-1} \\
& \times\left((I+R)\left(R^{2 N-j-1}+R^{2 N+j-1}-R^{2 N+j}-R^{2 N-j}+R^{j}-R^{j-2}\right)-2 R^{2 N+j-2}\right) \varphi_{j}, \\
J_{2}^{3}= & S_{\tau} \sum_{j=1}^{N} \rho\left(t_{j}-\frac{\tau}{2}\right) \frac{\tau}{2}\left(I-R^{2 N}\right)^{-1}(I-R)(I+R) R^{-1}\left(I-R^{2 N-2 j+1}\right) \\
& \times \sum_{i=1}^{j-1} R^{j-i}\left(I-R^{2 i}\right)(I+R)^{-1}\left(\varphi_{i}-\varphi_{j}\right)=J_{2}^{3,1}+J_{2}^{3,2}, \\
J_{2}^{3,1}= & S_{\tau} \sum_{j=1}^{\left[\frac{N}{2}\right]} \rho\left(t_{j}-\frac{\tau}{2}\right) \frac{\tau}{2}\left(I-R^{2 N}\right)^{-1}(I-R)(I+R) R^{-1}\left(I-R^{2 N-2 j+1}\right) \\
& \times \sum_{i=1}^{j-1} R^{j-i}\left(I-R^{2 i}\right)(I+R)^{-1}\left(\varphi_{i}-\varphi_{j}\right), \\
J_{2}^{3,2}= & S_{\tau} \sum_{j=\left[\frac{N}{2}\right]+1}^{N} \rho\left(t_{j}-\frac{\tau}{2}\right) \frac{\tau}{2}\left(I-R^{2 N}\right)^{-1}(I-R)(I+R) R^{-1}\left(I-R^{2 N-2 j+1}\right) \\
& \times \sum_{i=j+1}^{N-1} R^{i-j}\left(I-R^{2 N-2 i}\right)(I+R)^{-1}\left(\varphi_{i}-\varphi_{j}\right) . \\
& \times \sum_{i=1}^{j-1} R^{j-i}\left(I-R^{2 i}\right)(I+R)^{-1}\left(\varphi_{i}-\varphi_{j}\right), \\
& \times \sum_{i=j+1}^{4,2}=S_{\tau} \sum_{j=1}^{N-1} \rho\left(t_{j}-\frac{\tau}{2}\right) \frac{\tau}{2}\left(I-R^{2 N}\right)^{-1}(I-R)(I+R)\left(I-R^{2 j-1}\right) \\
J_{2}^{4}= & S_{\tau} \sum_{j=1}^{N} \rho\left(t_{j}-\frac{\tau}{2}\right) \frac{\tau}{2}\left(I-R^{2 N}\right)^{-1}(I-R)(I+R)\left(I-R^{2 j-1}\right) \\
& \times \sum_{i=j+1}^{N-1} R^{i-j}\left(I-R^{2 N-2 i}\right)(I+R)^{-1}\left(\varphi_{i}-\varphi_{j}\right)=J_{2}^{4,1}+J_{2}^{4,2}, \\
& \left.\sum^{2 N-2 i}\right)(I+R)^{-1}\left(\varphi_{i}-\varphi_{j}\right), \\
& \\
&
\end{aligned}
$$


Second, let us estimate $J_{2}^{m}$ for any $m=2, \ldots, 4$ separately. We start with $J_{2}^{2}$. Using estimates (4), (5) and the definition of the norm of the space $C_{01}^{\alpha}\left([0,1]_{\tau}, H\right)$, we get

$$
\begin{aligned}
\left\|J_{2}^{2}\right\|_{H} \leq & \left\|S_{\tau}\right\|_{H \rightarrow H} \sum_{j=1}^{N}\left|\rho\left(t_{j}-\frac{\tau}{2}\right)\right| \frac{\tau}{2}\left\|\left(I-R^{2 N}\right)^{-1}\right\|_{H \rightarrow H} \\
& \times\|I-R\|_{H \rightarrow H}\left\|(I+R)^{-1}\right\|_{H \rightarrow H}\left(\| I + R \| _ { H \rightarrow H } \left(\left\|R^{2 N-j-1}\right\|_{H \rightarrow H}\right.\right. \\
& \left.+\left\|R^{2 N+j-1}\right\|_{H \rightarrow H}+\left\|R^{2 N+j}\right\|_{H \rightarrow H}+\left\|R^{2 N-j}\right\|_{H \rightarrow H}+\left\|R^{j}\right\|_{H \rightarrow H}+\left\|R^{j-2}\right\|_{H \rightarrow H}\right) \\
& \left.+\left\|R^{2 N+j-2}\right\|_{H \rightarrow H}\right)\left\|\varphi_{j}\right\|_{H} \\
\leq & M_{1}(\delta) \sum_{j=1}^{N}\left|\rho\left(t_{j}-\frac{\tau}{2}\right)\right| \frac{\tau}{2} \max _{1 \leq j \leq N}\left\|\varphi_{j}\right\|_{H} \\
\leq & M_{1}(\delta) \sum_{j=1}^{N}\left|\rho\left(t_{j}-\frac{\tau}{2}\right)\right| \frac{\tau}{2}\left\|\varphi^{\tau}\right\|_{C_{01}^{\alpha}\left([0,1]_{\tau}, H\right)} .
\end{aligned}
$$

From (3) it follows that

$$
\left\|J_{2}^{2}\right\|_{H} \leq M_{2}(\delta)\left\|\varphi^{\tau}\right\|_{C_{01}^{\alpha}\left([0,1]_{\tau}, H\right)} .
$$

Now, let us estimate $J_{2}^{3,1}$. Using estimates (4), (5) and the definition of the norm of the space $C_{01}^{\alpha}\left([0,1]_{\tau}, H\right)$, we obtain

$$
\begin{aligned}
\left\|J_{2}^{3,1}\right\|_{H} \leq & \left\|S_{\tau}\right\|_{H \rightarrow H} \sum_{j=1}^{\left[\frac{N}{2}\right]}\left|\rho\left(t_{j}-\frac{\tau}{2}\right)\right| \frac{\tau}{2}\left\|\left(I-R^{2 N}\right)^{-1}\right\|_{H \rightarrow H} \\
& \times \sum_{i=1}^{j-1}\left\|R^{j-i}\left(I-R^{2 N-2 j+1}\right)(I-R)\right\|_{H \rightarrow H}\left\|I-R^{2 i}\right\|_{H \rightarrow H}\left\|R^{-1}\right\|_{H \rightarrow H}\left\|\varphi_{i}-\varphi_{j}\right\|_{H} \\
\leq & M(\delta) \sum_{j=1}^{\left[\frac{N}{2}\right]}\left|\rho\left(t_{j}-\frac{\tau}{2}\right)\right| \frac{\tau}{2} \sum_{i=1}^{j-1} \frac{\tau((j-i) \tau)^{\alpha}}{(j-i) \tau((N-i) \tau)^{\alpha}(j \tau)^{\alpha}}\left\|\varphi^{\tau}\right\|_{C_{01}^{\alpha}([0,1] \tau, H)} \\
\leq & M(\delta) \sum_{j=1}^{\left[\frac{N}{2}\right]} \frac{\left|\rho\left(t_{j}-\frac{\tau}{2}\right)\right| \tau}{2(j \tau)^{\alpha}((N-j) \tau)^{\alpha}} \sum_{i=1}^{j-1} \frac{\tau}{((j-i) \tau)^{1-\alpha}}\left\|\varphi^{\tau}\right\|_{C_{01}^{\alpha}\left([0,1]_{\tau}, H\right)} .
\end{aligned}
$$

The sum

$$
\sum_{i=1}^{j-1} \frac{\tau}{((j-i) \tau)^{1-\alpha}}
$$

is the lower Darboux integral sum for the integral

$$
\int_{0}^{j \tau} \frac{d s}{(j \tau-s)^{1-\alpha}}
$$

It follows that

$$
\left\|J_{2}^{3,1}\right\|_{H} \leq M(\delta) \sum_{j=1}^{\left[\frac{N}{2}\right]} \frac{\left|\rho\left(t_{j}-\frac{\tau}{2}\right)\right| \tau}{\alpha((N-j) \tau)^{\alpha}}\left\|\varphi^{\tau}\right\|_{C_{01}^{\alpha}\left([0,1]_{\tau}, H\right)} .
$$


By the lower Darboux integral sum for the integral it concludes that

$$
\left\|J_{2}^{3,1}\right\|_{H} \leq M(\delta) \frac{2^{\alpha-2}}{\alpha(1-\alpha)} \sum_{j=1}^{\left[\frac{N}{2}\right]}\left|\rho\left(t_{j}-\frac{\tau}{2}\right)\right| \tau\left\|\varphi^{\tau}\right\|_{C_{01}^{\alpha}([0,1] \tau, H)}
$$

For $J_{2}^{3,2}$, applying (4), (5) and the definition of the norm of the space $C_{01}^{\alpha}\left([0,1]_{\tau}, H\right)$, we get

$$
\begin{aligned}
\left\|J_{2}^{3,2}\right\|_{H} \leq & \left\|S_{\tau}\right\|_{H \rightarrow H} \sum_{j=\left[\frac{N}{2}\right]+1}^{N}\left|\rho\left(t_{j}-\frac{\tau}{2}\right)\right| \frac{\tau}{2}\left\|\left(I-R^{2 N}\right)^{-1}\right\|_{H \rightarrow H} \\
& \times \sum_{i=1}^{j-1}\left\|R^{j-i}\left(I-R^{2 N-2 j+1}\right)(I-R)\right\|_{H \rightarrow H}\left\|I-R^{2 i}\right\|_{H \rightarrow H}\left\|R^{-1}\right\|_{H \rightarrow H}\left\|\varphi_{i}-\varphi_{j}\right\|_{H} \\
\leq & M(\delta) \sum_{j=\left[\frac{N}{2}\right]+1}^{N}\left|\rho\left(t_{j}-\frac{\tau}{2}\right)\right| \frac{\tau}{2} \\
& \times \sum_{i=1}^{j-1} \frac{(2 \tau(N-j+1))^{\alpha}}{((j-i) \tau)^{1-\alpha}((2 N-j-i+1) \tau)^{\alpha}(j \tau)^{\alpha}((N-i) \tau)^{\alpha}}\left\|\varphi^{\tau}\right\|_{C_{01}^{\alpha}\left([0,1]_{\tau}, H\right)} \\
\leq & M(\delta) \sum_{j=\left[\frac{N}{2}\right]+1}^{N} \frac{\left|\rho\left(t_{j}-\frac{\tau}{2}\right)\right| \tau 2^{\alpha}((N-j+1) \tau)^{\alpha}}{((N-j) \tau)^{\alpha}(j \tau)^{\alpha}} \\
& \times \sum_{i=1}^{j-1} \frac{\tau}{((j-i) \tau)^{1-\alpha}((N-j-i+N+1) \tau)^{\alpha}}\left\|\varphi^{\tau}\right\|_{C_{01}^{\alpha}\left([0,1]_{\tau}, H\right)^{\prime}} .
\end{aligned}
$$

The sum

$$
\sum_{i=1}^{j-1} \frac{\tau}{((j-i) \tau)^{1-\alpha}((N-j-i+N+1) \tau)^{\alpha}}
$$

is the lower Darboux integral sum for the integral

$$
\int_{0}^{j \tau} \frac{d s}{(j \tau-s)^{1-\alpha}(N \tau-j \tau-s+\tau+N \tau)^{\alpha}}
$$

Since

$$
\begin{aligned}
& \int_{0}^{j \tau} \frac{d s}{(j \tau-s)^{1-\alpha}(N \tau-j \tau-s+N \tau+\tau)^{\alpha}} \\
& \quad \leq \frac{1}{(N \tau-j \tau+\tau)^{\alpha}} \int_{0}^{j \tau} \frac{d s}{(j \tau-s)^{1-\alpha}} \leq \frac{M}{\alpha(j \tau)^{\alpha}},
\end{aligned}
$$

it follows that

$$
\left\|J_{2}^{3,2}\right\|_{H} \leq M(\delta) \sum_{j=\left[\frac{N}{2}\right]+1}^{N}\left|\rho\left(t_{j}-\frac{\tau}{2}\right)\right| \tau \frac{2^{\alpha}}{(j \tau)^{\alpha}(N \tau-j \tau+\tau)^{\alpha} \alpha(j \tau)^{-\alpha}}\left\|\varphi^{\tau}\right\|_{C_{01}^{\alpha}([0,1] \tau, H} .
$$


By the lower Darboux integral sum for the integral it follows that

$$
\left\|J_{2}^{3,2}\right\|_{H} \leq \frac{M(\delta) 2^{2 \alpha-2}}{\alpha(1-\alpha)} \sum_{j=\left[\frac{N}{2}\right]+1}^{N}\left|\rho\left(t_{j}-\frac{\tau}{2}\right)\right| \tau\left\|\varphi^{\tau}\right\|_{C_{01}^{\alpha}\left([0,1]_{\tau}, H\right)}
$$

Combining $J_{2}^{3,1}$ and $J_{2}^{3,2}$, we get

$$
\left\|J_{2}^{3}\right\|_{H} \leq \frac{M_{3}(\delta)}{\alpha(1-\alpha)} \sum_{j=1}^{N}\left|\rho\left(t_{j}-\frac{\tau}{2}\right)\right| \tau\left\|\varphi^{\tau}\right\|_{C_{01}^{\alpha}\left([0,1]_{\tau}, H\right)}
$$

From (3) it follows that

$$
\left\|J_{2}^{3}\right\|_{H} \leq \frac{M_{4}(\delta)}{\alpha(1-\alpha)}\left\|\varphi^{\tau}\right\|_{C_{01}^{\alpha}\left([0,1]_{\tau}, H\right)}
$$

Next, let us estimate $J_{2}^{4,1}$. Using the estimates (4), (5), and the definition of the norm space $C_{01}^{\alpha}\left([0,1]_{\tau}, H\right)$, we obtain

$$
\begin{aligned}
\left\|J_{2}^{4,1}\right\|_{H} \leq & \left\|S_{\tau}\right\|_{H \rightarrow H} \sum_{j=1}^{\left[\frac{N}{2}\right]}\left|\rho\left(t_{j}-\frac{\tau}{2}\right)\right| \frac{\tau}{2}\left\|\left(I-R^{2 N}\right)^{-1}\right\|_{H \rightarrow H}\left\|I-R^{2 j-1}\right\|_{H \rightarrow H} \\
& \times \sum_{i=j+1}^{N-1}\left\|R^{i-j}\left(I-R^{2 N-2 i}\right)(I-R)\right\|_{H \rightarrow H}\left\|\varphi_{i}-\varphi_{j}\right\|_{H} \\
\leq & M(\delta) \sum_{j=1}^{\left[\frac{N}{2}\right]}\left|\rho\left(t_{j}-\frac{\tau}{2}\right)\right| \frac{\tau}{2} \\
& \times \sum_{i=j+1}^{N-1} \frac{2^{\alpha}((i-j) \tau)^{\alpha}(N \tau-i \tau)^{\alpha}}{((i-j) \tau)((N-j) \tau)^{\alpha}(i \tau)^{\alpha}((2 N-j-i) \tau)^{\alpha}}\left\|\varphi^{\tau}\right\|_{C_{01}^{\alpha}\left([0,1]_{\tau}, H\right)} \\
\leq & M(\delta) \sum_{j=1}^{\left[\frac{N}{2}\right]}\left|\rho\left(t_{j}-\frac{\tau}{2}\right)\right| \tau \frac{(N \tau-j \tau)^{\alpha}}{2(N \tau-j \tau)^{\alpha}(j \tau)^{\alpha}} \\
& \times \sum_{i=j+1}^{N-1} \frac{\tau}{((i-j) \tau)^{1-\alpha}((N-j-i+N) \tau)^{\alpha}}\left\|\varphi^{\tau}\right\|_{C_{01}^{\alpha}\left([0,1]_{\tau}, H\right)^{*}}
\end{aligned}
$$

The sum

$$
\sum_{i=j+1}^{N-1} \frac{\tau}{((i-j) \tau)^{1-\alpha}}
$$

is the lower Darboux integral sum for the integral

$$
\int_{j \tau}^{1} \frac{d s}{(s-j \tau)^{1-\alpha}}
$$


Since

$$
\int_{j \tau}^{1} \frac{d s}{(s-j \tau)^{1-\alpha}(2 N \tau-j \tau-s)^{\alpha}} \leq \frac{1}{(N \tau-j \tau)^{\alpha}} \int_{j \tau}^{1} \frac{d s}{(s-j \tau)^{1-\alpha}} \leq \frac{(N \tau-j \tau)^{\alpha}}{\alpha(N \tau-j \tau)^{\alpha}}
$$

it follows that

$$
\left\|J_{2}^{4,1}\right\|_{H} \leq M(\delta) \sum_{j=1}^{\left[\frac{N}{2}\right]} \frac{\left|\rho\left(t_{j}-\frac{\tau}{2}\right)\right| \tau}{(j \tau)^{\alpha} \alpha}\left\|\varphi^{\tau}\right\|_{C_{01}^{\alpha}\left([0,1]_{\tau}, H\right)} .
$$

By the lower Darboux integral sum for the integral it follows that

$$
\left\|J_{2}^{4,1}\right\|_{H} \leq \frac{M(\delta) 2^{2 \alpha-1}}{\alpha(1-\alpha)} \sum_{j=1}^{\left[\frac{N}{2}\right]}\left|\rho\left(t_{j}-\frac{\tau}{2}\right)\right| \tau\left\|\varphi^{\tau}\right\|_{C_{01}^{\alpha}\left([0,1]_{\tau}, H\right)}
$$

Finally, let us estimate $J_{2}^{4,2}$. Using the estimates (4), (5), and the definition of the norm space $C_{01}^{\alpha}\left([0,1]_{\tau}, H\right)$, we get

$$
\begin{aligned}
\left\|J_{2}^{4,2}\right\|_{H} \leq & \left\|S_{\tau}\right\|_{H \rightarrow H} \sum_{j=\left[\frac{N}{2}\right]+1}^{N}\left|\rho\left(t_{j}-\frac{\tau}{2}\right)\right| \frac{\tau}{2}\left\|\left(I-R^{2 N}\right)^{-1}\right\|_{H \rightarrow H}\left\|I-R^{2 j-1}\right\|_{H \rightarrow H} \\
& \times \sum_{i=j+1}^{N-1}\left\|R^{i-j}\left(I-R^{2 N-2 i}\right)(I-R)\right\|_{H \rightarrow H}\left\|\varphi_{i}-\varphi_{j}\right\|_{H} \\
\leq & M(\delta) \sum_{j=\left[\frac{N}{2}\right]+1}^{N}\left|\rho\left(t_{j}-\frac{\tau}{2}\right)\right| \frac{\tau}{2} \\
& \times \sum_{i=j+1}^{N-1} \frac{\tau((i-j) \tau)^{\alpha}}{((i-j) \tau)((N-i) \tau)^{\alpha}(i \tau)^{\alpha}}\left\|\varphi^{\tau}\right\|_{C_{01}^{\alpha}\left([0,1]_{\tau}, H\right)} \\
\leq & M(\delta) \sum_{j=\left[\frac{N}{2}\right]+1}^{N} \frac{\left|\rho\left(t_{j}-\frac{\tau}{2}\right)\right| \tau}{2((N-j) \tau)^{\alpha}(j \tau)^{\alpha}} \sum_{i=j+1}^{N-1} \frac{\tau}{((i-j) \tau)^{1-\alpha}}\left\|\varphi^{\tau}\right\|_{C_{01}^{\alpha}\left([0,1]_{\tau}, H\right)} .
\end{aligned}
$$

The sum

$$
\sum_{i=j+1}^{N-1} \frac{\tau}{((i-j) \tau)^{1-\alpha}}
$$

is the lower Darboux integral sum for the integral

$$
\int_{j \tau}^{1} \frac{d s}{(s-j \tau)^{1-\alpha}}
$$

Thus, we show that

$$
\left\|J_{2}^{4,2}\right\|_{H} \leq M(\delta) \sum_{j=\left[\frac{N}{2}\right]+1}^{N} \frac{\left|\rho\left(t_{j}-\frac{\tau}{2}\right)\right| \tau}{(j \tau)^{\alpha} \alpha}\left\|\varphi^{\tau}\right\|_{C_{01}^{\alpha}\left([0,1]_{\tau}, H\right)} .
$$


By the lower Darboux integral sum for the integral it follows that

$$
\left\|J_{2}^{4,2}\right\|_{H} \leq M(\delta) \frac{2^{\alpha-2}}{\alpha(1-\alpha)} \sum_{j=\left[\frac{N}{2}\right]+1}^{N}\left|\rho\left(t_{j}-\frac{\tau}{2}\right)\right| \tau\left\|\varphi^{\tau}\right\|_{C_{01}^{\alpha}\left([0,1]_{\tau}, H\right)}
$$

Combining $J_{2}^{4,1}$ and $J_{2}^{4,2}$, we get

$$
\left\|J_{2}^{4}\right\|_{H} \leq M(\delta)\left(\frac{2^{\alpha-2}}{\alpha(1-\alpha)}+\frac{2^{2 \alpha-1}}{\alpha(1-\alpha)}\right) \sum_{j=1}^{N}\left|\rho\left(t_{j}-\frac{\tau}{2}\right)\right| \tau\left\|\varphi^{\tau}\right\|_{C_{01}^{\alpha}\left([0,1]_{\tau}, H\right)} .
$$

From (3) it follows that

$$
\left\|J_{2}^{4}\right\|_{H} \leq \frac{M_{5}(\delta)}{\alpha(1-\alpha)}\left\|\varphi^{\tau}\right\|_{C_{01}^{\alpha}\left([0,1]_{\tau}, H\right)}
$$

Combining estimates for $J_{2}^{m}, m=2, \ldots, 4$ we get the estimate (19). Theorem 5 is proved.

\section{Applications}

Now, the application of Theorems 3-5 will be given. First, we consider the mixed boundary-value problem for elliptic equation

$$
\left\{\begin{array}{l}
-u_{t t}-\left(a(x) u_{x}\right)_{x}+\delta u=f(t, x), \quad 0<t<1,0<x<1, \\
u(t, 0)=u(t, 1), \quad u_{x}(t, 1)=u_{x}(t, 0), \quad 0 \leq t \leq 1, \\
u(0, x)=\varphi(x), \quad u(1, x)=\int_{0}^{1} \rho(\lambda) u(\lambda, x) d \lambda+\psi(x), \quad 0 \leq x \leq 1,
\end{array}\right.
$$

where $a(x), \varphi(x), \psi(x)$ and $f(t, x)$ are given sufficiently smooth functions and $a(x) \geq a>0$, $a(1)=a(0), \delta=$ const $>0$. The discretization of problem (21) is carried out in two steps. In the first step, let us define the grid space

$$
[0,1]_{h}=\left\{x: x_{n}=n h, 0 \leq n \leq M, M h=1\right\} .
$$

We introduce the Hilbert space $L_{2 h}=L_{2}\left([0,1]_{h}\right)$ of the grid functions $\varphi^{h}(x)=\left\{\varphi_{n}\right\}_{n=1}^{M-1}$ defined on $[0,1]_{h}$, equipped with the norms

$$
\begin{aligned}
& \left\|\varphi^{h}\right\|_{L_{2 h}}=\left(\sum_{x \in[0,1]_{h}}\left|\varphi^{h}(x)\right|^{2} h\right)^{\frac{1}{2}}, \\
& \left\|\varphi^{h}\right\|_{W_{2 h}^{2}}=\left\|\varphi^{h}\right\|_{L_{2 h}}+\left(\sum_{x \in[0,1]_{h}}\left|\left(\varphi^{h}(x)\right)_{x}\right|^{2} h\right)^{1 / 2}+\left(\sum_{x \in[0,1]_{h}}\left|\left(\varphi^{h}(x)\right)_{\bar{x}, x}\right|^{2} h\right)^{1 / 2} .
\end{aligned}
$$

To the differential operator $A$ generated by the problem (21) we assign the difference operator $A_{h}^{x}$ by the formula

$$
A_{h}^{x} \varphi^{h}(x)=\left\{-\left(a(x) \varphi_{\bar{x}}\right)_{x, n}+\delta \varphi_{n}\right\}_{1}^{M-1}
$$


acting in the space of the grid functions $\varphi^{h}(x)=\left\{\varphi_{n}\right\}_{1}^{M-1}$ satisfying the conditions $\varphi_{0}=\varphi_{M}$, $\varphi_{1}-\varphi_{0}=\varphi_{M}-\varphi_{M-1}$. It is know that $A_{h}^{x}$ is a self-adjoint positive definite operator in $L_{2 h}$. With the help of $A_{h}^{x}$, we arrive at the nonlocal boundary-value problem

$$
\left\{\begin{array}{l}
-\frac{d^{2} u^{h}(t, x)}{d t^{2}}+A_{h}^{x} u^{h}(t, x)=f^{h}(t, x), \quad 0<t<1, x \in[0,1]_{h}, \\
u^{h}(0, x)=\varphi^{h}(x) ; \quad u^{h}(1, x)=\int_{0}^{1} \rho(t) u^{h}(t, x) d t+\psi^{h}(x), \quad x \in[0,1]_{h}
\end{array}\right.
$$

for an infinite system of ordinary differential equations. Therefore, in the second step, equation (23) is replaced by the difference scheme (2), and we get the following difference scheme:

$$
\left\{\begin{array}{l}
-\frac{u_{k+1}^{h}(x)-2 u_{k}^{h}(x)+u_{k-1}^{h}(x)}{\tau^{2}}+A_{h}^{x} u_{k}^{h}(x)=\varphi_{k}^{h}(x), \\
\quad \varphi_{k}^{h}(x)=f^{h}\left(t_{k}, x\right), t_{k}=k \tau, 1 \leq k \leq N-1, N \tau=1, x \in[0,1]_{h}, \\
u_{0}^{h}(x)=\varphi^{h}(x) ; \quad u_{N}^{h}(x)=\sum_{j=1}^{N} \rho\left(t_{j}-\frac{\tau}{2}\right) \tau\left(\frac{u_{j}^{h}(x)+u_{j-1}^{h}(x)}{2}\right)+\psi^{h}(x), \quad x \in[0,1]_{h}
\end{array}\right.
$$

for the numerical solution of (21).

Theorem 6 Let $\tau$ and $h$ be sufficiently small positive numbers. Then under the assumption (3), the solution of the difference scheme (24) satisfies the following stability and almost coercivity estimates:

$$
\begin{aligned}
& \max _{1 \leq k \leq N-1}\left\|u_{k}^{h}\right\|_{L_{2 h}} \leq M(\delta)\left[\max _{1 \leq k \leq N-1}\left\|\varphi_{k}^{h}\right\|_{L_{2 h}}+\left\|\psi^{h}\right\|_{L_{2 h}}+\left\|\varphi^{h}\right\|_{L_{2 h}}\right], \\
& \max _{1 \leq k \leq N-1}\left\|\tau^{-2}\left(u_{k+1}^{h}-2 u_{k}^{h}+u_{k-1}^{h}\right)\right\|_{L_{2 h}}+\max _{1 \leq k \leq N-1}\left\|\left(u_{k}^{h}\right)\right\|_{W_{2 h}^{2}} \\
& \quad \leq M(\delta)\left[\ln \frac{1}{\tau+|h|} \max _{1 \leq k \leq N-1}\left\|\varphi_{k}^{h}\right\|_{L_{2 h}}+\left\|\varphi^{h}\right\|_{W_{2 h}^{2}}+\left\|\psi^{h}\right\|_{W_{2 h}^{2}}\right] .
\end{aligned}
$$

The proof of Theorem 6 is based on Theorems 3 and 4, on the estimate

$$
\min \left\{\ln \frac{1}{\tau}, 1+\left|\ln \left\|B_{h}^{x}\right\|_{L_{2 h} \rightarrow L_{2 h}}\right|\right\} \leq M \ln \frac{1}{\tau+|h|},
$$

and on the symmetry properties of the difference operator $A_{h}^{x}$ defined by the formula (22) in $L_{2 h}$.

Theorem 7 Let $\tau$ and $|h|$ be sufficiently small positive numbers. Then under the assumption (3), the solution of the difference scheme (24) satisfies the following coercivity estimate:

$$
\begin{aligned}
& \left\|\left\{\tau^{-2}\left(u_{k+1}^{h}-2 u_{k}^{h}+u_{k-1}^{h}\right)\right\}_{1}^{N-1}\right\|_{C_{01}^{\alpha}\left([0,1]_{\tau}, L_{2 h}\right)}+\left\|\left\{u_{k}^{h}\right\}_{1}^{N-1}\right\|_{C_{01}^{\alpha}\left([0,1]_{\tau}, W_{2 h}^{2}\right)} \\
& \quad \leq M(\delta)\left[\left\|\varphi^{h}\right\|_{W_{2 h}^{2}}+\left\|\psi^{h}\right\|_{W_{2 h}^{2}}+\frac{1}{\alpha(1-\alpha)}\left\|\left\{\varphi_{k}^{h}\right\}_{1}^{N-1}\right\|_{C_{01}^{\alpha}\left([0,1]_{\tau}, L_{2 h}\right)}\right] .
\end{aligned}
$$

The proof of Theorem 7 is based on Theorem 5 and the symmetry properties of the difference operator $A_{h}^{x}$ defined by formula (22).

Second, let $\Omega$ be the unit open cube in $\mathbb{R}^{n}\left(x=\left(x_{1}, \ldots, x_{n}\right): 0<x_{k}<1,1 \leq k \leq n\right)$ with boundary $S, \bar{\Omega}=\Omega \cup S$. In $[0,1] \times \Omega$, the Dirichlet-Bitsadze-Samarskii type mixed 
boundary-value problem for the multidimensional elliptic equation

$$
\left\{\begin{array}{l}
-u_{t t}-\sum_{r=1}^{n}\left(a_{r}(x) u_{x_{r}}\right)_{x_{r}}=f(t, x), \quad 0<t<1, x=\left(x_{1}, \ldots, x_{n}\right) \in \Omega, \\
u(0, x)=\varphi(x), \quad u(1, x)=\int_{0}^{1} \rho(\lambda) u(\lambda, x) d \lambda+\psi(x), \quad x \in \bar{\Omega}, \\
\left.u(t, x)\right|_{x \in S}=0, \quad x \in \bar{\Omega}
\end{array}\right.
$$

is considered. We will study the problem (26) under the assumption (3). Here, $a_{r}(x)$ ( $x \in$ $\Omega), \psi(x), \varphi(x)(x \in \bar{\Omega})$ and $f(t, x)(t \in(0,1), x \in \Omega)$ are smooth functions and $a_{r}(x) \geq a>0$. The discretization of problem (26) is carried out in two steps. In the first step let us define the grid sets

$$
\begin{gathered}
\bar{\Omega}_{h}=\left\{x=x_{m}=\left(h_{1} m_{1}, \ldots, h_{m} m_{m}\right), m=\left(m_{1}, \ldots, m_{m}\right), 0 \leq m_{r} \leq N_{r},\right. \\
\left.h_{r} N_{r}=1, r=1, \ldots, m\right\}, \quad \Omega_{h}=\widetilde{\Omega}_{h} \cap \Omega, \quad S_{h}=\widetilde{\Omega}_{h} \cap S .
\end{gathered}
$$

We introduce the Hilbert space $L_{2 h}=L_{2}\left(\widetilde{\Omega}_{h}\right)$ of the grid functions $\varphi^{h}(x)=\left\{\varphi\left(h_{1} m_{1}, \ldots\right.\right.$, $\left.h_{m} m_{m}\right)$ \} defined on $\widetilde{\Omega}_{h}$, equipped with the norms

$$
\begin{aligned}
\left\|\varphi^{h}\right\|_{L_{2 h}}= & \left(\sum_{x \in \bar{\Omega}_{h}}\left|\varphi^{h}(x)\right|^{2} h_{1} \cdots h_{m}\right)^{1 / 2}, \\
\left\|\varphi^{h}\right\|_{W_{2 h}^{2}}= & \left\|\varphi^{h}\right\|_{L_{2 h}}+\left(\sum_{x \in \tilde{\Omega}_{h}} \sum_{r=1}^{m}\left|\left(\varphi^{h}(x)\right)_{x_{r}}\right|^{2} h_{1} \cdots h_{m}\right)^{1 / 2} \\
& +\left(\sum_{x \in \tilde{\Omega}_{h}} \sum_{r=1}^{m}\left|\left(\varphi^{h}(x)\right)_{x_{r} \bar{x}_{r}, m_{r}}\right|^{2} h_{1} \cdots h_{m}\right)^{1 / 2} .
\end{aligned}
$$

To the differential operator $A$ generated by the problem (26), we assign the difference operator $A_{h}^{x}$ by the formula

$$
A_{h}^{x} u^{h}(x)=-\sum_{r=1}^{m}\left(a_{r}(x) u_{\bar{x}_{r}}^{h}\right)_{x_{r}, j_{r}},
$$

acting in the space of the grid functions $u^{h}(x)$, satisfying the conditions $u^{h}(x)=0$ for all $x \in S_{h}$. It is known that $A_{h}^{x}$ is a self-adjoint positive definite operator in $L_{2 h}$. With the help of $A_{h}^{x}$, we arrive at the nonlocal boundary-value problem for an infinite system of ordinary differential equations

$$
\left\{\begin{array}{l}
-\frac{d^{2} u^{h}(t, x)}{d t^{2}}+A_{h}^{x} u^{h}(t, x)=f^{h}(t, x), \quad 0<t<1, x \in \widetilde{\Omega}_{h}, \\
u^{h}(0, x)=\varphi^{h}(x) ; \quad u^{h}(1, x)=\int_{0}^{1} \rho(t) u^{h}(t, x) d t+\psi^{h}(x), \quad x \in \widetilde{\Omega}_{h} .
\end{array}\right.
$$

In the second step, (28) is replaced by the difference scheme (2), and we get the following difference scheme:

$$
\left\{\begin{array}{l}
-\frac{u_{k+1}^{h}(x)-2 u_{k}^{h}(x)+u_{k-1}^{h}(x)}{\tau^{2}}+A_{h}^{x} u_{k}^{h}(x)=\varphi_{k}^{h}(x), \\
\varphi_{k}^{h}(x)=f^{h}\left(t_{k}, x\right), x \in \Omega_{h}, t_{k}=k \tau, 1 \leq k \leq N-1, N \tau=1, \\
u_{0}^{h}(x)=\varphi^{h}(x), \quad x \in \widetilde{\Omega}_{h}, \\
u_{N}^{h}(x)=\sum_{j=1}^{N} \rho\left(t_{j}-\frac{\tau}{2}\right) \tau\left(\frac{u_{j}^{h}(x)+u_{j-1}^{h}(x)}{2}\right)+\psi^{h}(x), \quad x \in \widetilde{\Omega}_{h}
\end{array}\right.
$$

for the numerical solution of (26). 
Theorem 8 Let $\tau$ and $|h|=\sqrt{h_{1}^{2}+\cdots+h_{n}^{2}}$ be sufficiently small positive numbers. Then under the assumption (3) the solution of the difference scheme (29) satisfies the following stability estimates:

$$
\max _{1 \leq k \leq N-1}\left\|u_{k}^{h}\right\|_{L_{2 h}} \leq M(\delta)\left[\max _{1 \leq k \leq N-1}\left\|\varphi_{k}^{h}\right\|_{L_{2 h}}+\left\|\psi^{h}\right\|_{L_{2 h}}+\left\|\varphi^{h}\right\|_{L_{2 h}}\right] .
$$

The proof of Theorem 8 is based on Theorem 3 and the symmetry properties of the difference operator $A_{h}^{x}$ defined by (27) in $L_{2 h}$.

Theorem 9 Let $\tau$ and $|h|$ be sufficiently small positive numbers. Then under the assumption (3) the solution of the difference scheme (29) satisfies the following almost coercivity estimates:

$$
\begin{aligned}
& \max _{1 \leq k \leq N-1}\left\|\tau^{-2}\left(u_{k+1}^{h}-2 u_{k}^{h}+u_{k-1}^{h}\right)\right\|_{L_{2 h}}+\max _{1 \leq k \leq N-1}\left\|u_{k}^{h}\right\|_{W_{2 h}^{2}} \\
& \quad \leq M(\delta)\left[\ln \frac{1}{\tau+|h|} \max _{1 \leq k \leq N-1}\left\|\varphi_{k}^{h}\right\|_{L_{2 h}}+\left\|\varphi^{h}\right\|_{W_{2 h}^{2}}+\left\|\psi^{h}\right\|_{W_{2 h}^{2}}\right] .
\end{aligned}
$$

The proof of Theorem 9 is based on Theorem 4, on the estimate (25), on the symmetry properties of the difference operator $A_{h}^{x}$ defined by (27) in $L_{2 h}$, and on the following theorem on the coercivity inequality for the solution of the elliptic difference problem in $L_{2 h}$.

Theorem 10 For the solutions of the elliptic difference problem

$$
A_{h}^{x} u^{h}(x)=\omega^{h}(x), \quad x \in \Omega_{h}, \quad u^{h}(x)=0, \quad x \in S_{h}
$$

the following coercivity inequality holds [30]:

$$
\left\|u^{h}\right\|_{W_{2 h}^{2}} \leq M(\delta)\left\|\omega^{h}\right\|_{L_{2 h}} .
$$

Theorem 11 Let $\tau$ and $|h|$ be sufficiently small positive numbers. Then under the assumption (3) the solution of the difference scheme (29) satisfies the following coercivity stability estimate:

$$
\begin{aligned}
& \left\|\left\{\tau^{-2}\left(u_{k+1}^{h}-2 u_{k}^{h}+u_{k-1}^{h}\right)\right\}_{1}^{N-1}\right\|_{C_{01}^{\alpha}\left([0,1]_{\tau}, L_{2 h}\right)}+\left\|\left\{u_{k}^{h}\right\}_{1}^{N-1}\right\|_{C_{01}^{\alpha}\left([0,1]_{\tau}, W_{2 h}^{2}\right)} \\
& \leq M(\delta)\left[\left\|\varphi^{h}\right\|_{W_{2 h}^{2}}+\left\|\psi^{h}\right\|_{W_{2 h}^{2}}+\frac{1}{\alpha(1-\alpha)}\left\|\left\{\varphi_{k}^{h}\right\}_{1}^{N-1}\right\|_{C_{01}^{\alpha}\left([0,1]_{\tau} L_{2 h}\right)}\right] .
\end{aligned}
$$

The proof of Theorem 11 is based on Theorem 5, on the symmetry properties of the difference operator $A_{h}^{x}$ defined by the formula (27), and on Theorem 10 on the coercivity inequality for the solution of the elliptic difference equation in $L_{2 h}$.

\section{Numerical results}

We consider the Bitsadze -Samarskii type nonlocal boundary problem for the elliptic equation

$$
\left\{\begin{array}{l}
-\frac{\partial^{2} u(t, x)}{\partial t^{2}}-\frac{\partial^{2} u(t, x)}{\partial x^{2}}+u=\pi^{2} \exp (-t) \sin (\pi x), \\
\quad 0<t<1,0<x<1, \quad u(0, x)=\sin (\pi x), \\
u(1, x)=\int_{0}^{1} e^{-\lambda} u(\lambda, x) d \lambda+\left(\exp (-1)+\frac{1}{2} \exp (-2)-\frac{1}{2}\right) \sin (\pi x), \quad 0<x<1, \\
u(t, 0)=u(t, 1)=0, \quad 0<t<1 .
\end{array}\right.
$$


Table 1 The errors for first- and second-order difference scheme

\begin{tabular}{llll}
\hline & $\mathbf{N}=\boldsymbol{M}=\mathbf{2 0}$ & $\mathbf{N}=\boldsymbol{M}=\mathbf{4 0}$ & $\mathbf{N =} \boldsymbol{M = 8 0}$ \\
\hline First-order difference scheme & 0.173 & 0.0087 & 0.0044 \\
Second-order difference scheme & $6.245 \mathrm{e}-004$ & $1.562 \mathrm{e}-004$ & $3.906 \mathrm{e}-005$ \\
\hline
\end{tabular}

The exact solution of this problem is

$$
u(t, x)=\exp (-t) \sin (\pi x) .
$$

In the present part for the approximate solutions of the Bitsadze-Samarskii type nonlocal boundary-value problem (30), we will use the first and second orders of the accuracy difference schemes with grid intervals $\tau=\frac{1}{N}, h=\frac{1}{M}$ for $t$ and $x$, respectively. For the approximate solution of the nonlocal boundary Bitsadze-Samarskii type problem (30), we consider the set $[0,1]_{\tau} \times[0,1]_{h}$ of a family of grid points depending on the small parameters $\tau$ and $h$,

$$
\begin{aligned}
{[0,1]_{\tau} \times[0,1]_{h}=} & \left\{\left(t_{k}, x_{n}\right): t_{k}=k \tau, 1 \leq k \leq N-1, N \tau=1,\right. \\
& \left.x_{n}=n h, 1 \leq n \leq M-1, M h=1\right\} .
\end{aligned}
$$

Applying the first order of the accuracy difference scheme from [31] and the second order of the accuracy difference scheme (2) for the approximate solutions of the problem, we have the second-order difference equations with respect to $n$ with matrix coefficients. To solve these difference equations, we have applied the procedure of a modified Gauss elimination method for the difference equations with respect to $n$ with matrix coefficients. To obtain the solution of (2), we use MATLAB programming. The errors are computed by

$$
E_{M}^{N}=\max _{1 \leq k \leq N-1}\left(\sum_{n=1}^{M-1}\left|u\left(t_{k}, x_{n}\right)-u_{n}^{k}\right|^{2} h\right)^{\frac{1}{2}}
$$

of numerical solutions for different values of $M$ and $N$, where $u\left(t_{k}, x_{n}\right)$ represents the exact solution and $u_{n}^{k}$ represents the numerical solution at $\left(t_{k}, x_{n}\right)$. The results are shown in Table 1 , respectively.

\section{Conclusion}

In this paper, the second order of the accuracy difference scheme for the approximate solution of the Bitsadze-Samarskii type nonlocal boundary-value problem with the integral condition for elliptic equations is presented. Theorems on the stability estimates, almost coercive stability estimates, and coercive stability estimates for the solution of difference scheme for elliptic equations are proved. The theoretical statements for the solution of this difference scheme are supported by the result of a numerical example. As can be seen from Table 1, the second order of the accuracy difference scheme is more accurate than the first order of the accuracy difference scheme. 


\section{Authors' contributions}

EO carried out the studies, participated in the sequence alignment and drafted the manuscript and AA carried out the studies, participated in the sequence alignment. All authors read and approved the final manuscript.

\section{Author details}

'Department of Mathematics, Fatih University, Istanbul, 34500, Turkey. ${ }^{2}$ Department of Econometrics, Canakkale Onsekiz Mart University, Canakkale, 17200, Turkey.

\section{Acknowledgements}

The authors would like to thank Prof. Dr. PE Sobolevskii for his helpful suggestions on the improvement of this paper.

Received: 11 October 2013 Accepted: 16 December 2013 Published: 13 Jan 2014

\section{References}

1. Bitsadze, AV, Samarskii, AA: On some simplest generalizations of linear elliptic problems. Dokl. Akad. Nauk SSSR 185(4), 739-740 (1969)

2. Samarskii, AA: Some problems in differential equation theory. Differ. Uravn. 16(11), 1925-1935 (1980)

3. Sapagovas, MP: Difference method of increased order of accuracy for the Poisson equation with nonlocal conditions. Differ. Equ. 44(7), 1018-1028 (2008)

4. Ashyralyev, A: A note on the Bitsadze-Samarskii type nonlocal boundary value problem in a Banach space. J. Math. Anal. Appl. 344(1), 557-573 (2008)

5. Ashyralyev, A, Ozturk, E: The numerical solution of Bitsadze-Samarskii nonlocal boundary value problems with the Dirichlet-Neumann condition. Abstr. Appl. Anal. 2012, 730804 (2012)

6. Ashyralyev, A, Ozturk, E: On a difference scheme of fourth-order of accuracy for the Bitsadze-Samarskii type nonlocal boundary value problem. Math. Methods Appl. Sci. 36(8), 936-955 (2013)

7. Ashyralyev, A, Ozturk, E: On Bitsadze-Samarskii type nonlocal boundary value problems for elliptic differential and difference equations: well-posedness. Appl. Math. Comput. 219(3), 1093-1107 (2012)

8. Volkova, EA, Dosiyev, AA, Buranay, SC: On the solution of a nonlocal problem. Comput. Math. Appl. 66(3), 330-338 (2013)

9. Ashyralyev, A, Tetikoğlu, FS: A note on Bitsadze-Samarskii type nonlocal boundary value problems: well-posedness. Numer. Funct. Anal. Optim. 34(9), 939-975 (2013)

10. Berikelashvili, G: On a nonlocal boundary value problem for a two-dimensional elliptic equation. Comput. Methods Appl. Math. 3(1), 35-44 (2003)

11. Gordeziani, DG: On a method of resolution of Bitsadze-Samarskii boundary value problem. Abst. Rep. Inst. Appl. Math. Tbilisi State Univ. 2, 38-40 (1970)

12. Kapanadze, DV: On the Bitsadze-Samarskii nonlocal boundary value problem. Differ. Equ. 23(3), $543-545$ (1987)

13. Il'in, VA, Moiseev, El: Two-dimensional nonlocal boundary value problems for Poisson's operator in differential and difference variants. Mat. Model. 1(2), 139-159 (1990)

14. Berikelashvili, GK: On the convergence rate of the finite-difference solution of a nonlocal boundary value problem for a second-order elliptic equation. Differ. Equ. 39(7), 945-953 (2003)

15. Skubaczewski, AL: Solvability of elliptic problems with Bitsadze-Samarskii boundary conditions. Differ. Uravn. 21(4), 701-706 (1985)

16. Ashyralyev, A, Tetikoğlu, FS: FDM for elliptic equations with Bitsadze-Samarskii-Dirichlet conditions. Abstr. Appl. Anal. 2012, 454831 (2012)

17. Ladyzhenskaya, OA, Ural'tseva, NN: Linear and Quasilinear Equations of Elliptic Type. Nauka, Moscow (1973) (Russian)

18. Gorbachuk, VL, Gorbachuk, ML: Boundary Value Problems for Differential-Operator Equations. Naukova Dumka, Kiev (1984) (Russian)

19. Grisvard, P: Elliptic Problems in Nonsmooth Domains. Pitman, London (1985)

20. Agmon, S: Lectures on Elliptic Boundary Value Problems. Van Nostrand, Princeton (1965)

21. Krein, SG: Linear Differential Equations in Banach Space. Nauka, Moscow (1966) (Russian)

22. Skubachevskii, AL: Elliptic Functional Differential Equations and Applications. Operator Theory - Advances and Applications. Birkhäuser, Basel (1997)

23. Agarwal, $R$, Bohner, $M$, Shakhmurov, VB: Maximal regular boundary value problems in Banach-valued weighted spaces. Bound. Value Probl. 18, 9-42 (2005)

24. Sobolevskii, PE: Well-posedness of difference elliptic equations. Discrete Dyn. Nat. Soc. 1(4), $219-231$ (1997)

25. Ashyralyev, A: Well-posed solvability of the boundary value problem for difference equations of elliptic type. Nonlinear Anal., Theory Methods Appl. 24(2), 251-256 (1995)

26. Ashyralyev, A, Altay, N: A note on the well-posedness of the nonlocal boundary value problem for elliptic difference equations. Appl. Math. Comput. 175(1), 49-60 (2006)

27. Agmon, S, Douglis, SA, Nirenberg, L: Estimates near the boundary for solutions of elliptic partial differential equations satisfying general boundary conditions II. Commun. Pure Appl. Math. 17, 35-92 (1964)

28. Sobolevskii, PE: On elliptic equations in a Banach space. Differ. Uravn. 4(7), 1346-1348 (1969) (Russian)

29. Ashyralyev, A, Sobolevskii, PE: New Difference Schemes for Partial Differential Equations. Birkhäuser, Basel (2004)

30. Sobolevskii, PE: On Difference Method for Approximate Solution of Differential Equations. Izdat. Voronezh. Gosud. Univ., Voronezh (1975)

31. Ozturk, E: Nonlocal boundary value problems for elliptic differential and difference equations. PhD thesis, Mathematics Department, Uludag University (2013) (Turkish)

10.1186/1687-2770-2014-14

Cite this article as: Ashyralyev and Ozturk: On a difference scheme of second order of accuracy for the

Bitsadze-Samarskii type nonlocal boundary-value problem. Boundary Value Problems 2014, 2014:14 